\title{
Myasthenia gravis: the unmet needs of a paradigmatic autoimmune disease
}

\author{
Pedro J Modrego $*, 1$ \\ ${ }^{1}$ Department of Neurology, Hospital Miguel Servet, Isabel la católica 1-3. Zaragoza -50009, Spain \\ *Author for correspondence: Fax: + 34976491 817; pmodrego@salud.aragon.es
}

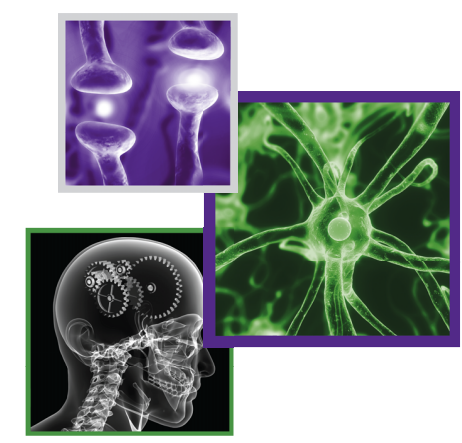

\section{"MG is a paradigmatic autoimmune disease, of chronic course, with exacerbations and remissions, even in the absence of treatment."}

First draft submitted: 7 April 2018; Accepted for publication: 9 April 2018; Published online: 26 June 2018

Keywords: autoantibodies $\bullet$ myasthenia gravis $\bullet$ treatment of refractory cases

Although new developments have arisen in the immunopathology and treatment of myasthenia gravis (MG) in the last decade, there are still many unmet needs. The origin of immunogenicity is not known, with around $10 \%$ of patients being seronegative for the antibodies ACh-R, musk-R and LRP4. Antititin antibodies have been associated to determined forms of MG with clinical and outcome implications. The role of the thymus and thymectomy warrants further research. With regard to treatment, monoclonal antibodies such as rituximab and eculizumab seem a reasonable alternative in refractory cases but the level of evidence needs improvement.

MG is a paradigmatic autoimmune disease, of chronic course, with exacerbations and remissions, even in the absence of treatment. The disease may occur at any age, it can be associated with other autoimmune diseases such as thyroid gland diseases and congenital forms are also possible. Pathophysiology is mainly characterized by the blockade of neuromuscular transmission at the site of the acetyl choline receptors, which results in fatigability and/or weakness of diverse muscles of the body, including the respiratory ones. The annual incidence ranges from 0.3 to 2.8/100,000 [1]. The clinical symptoms may appear acutely or in an insidious manner. In some cases, MG is detected immediately after a surgical intervention when the patient is weaned off the respiratory support. Since more than two decades ago, diagnosis has changed little in MG. With regard to treatment, new drugs are available but a number of unmet needs remain.

We know the antigens and antibodies present in the majority of patients (AChR, musk and LRPA), which are virtually diagnostic of MG but $10 \%$ of patients are triple seronegative. In this respect, antititin and antiagrin antibodies have arisen as a biomarker for these seronegative forms, but these antibodies may be also present in the AChR seropositive ones and whose clinical meaning is not clearly understood [2]. Among $667 \mathrm{MG}$ sera from 13 countries, $13.4 \%$ of triple seronegative MG patients were positive for antititin antibodies. An attempt of clinicalimmunological pattern has been reported. LRP4 antibodies were associated to late-onset myasthenia, with mild symptoms and good response to treatment. Anti-Agrin antibody MG was associated to early onset and moderate response to treatment. The patients negative for anti-AChR and positive for antititin presented with mild limb muscle weakness, whereas the patients that were positive for both anti-AChR and titin suffered from more severe MG with bulbar symptoms and myasthenic crisis [3]. However, the antibody titer does not correlate with the disease severity and is not indicative of better or worse outcome, so pathophysiologic mechanisms are beyond our knowledge.

The role of thymus in MG has not been elucidated yet. In some cases, the presence of hyperplasia or thymoma might be a consequence more than a pathogenic element. This assertion, supported by the fact of spontaneous regression of thymomas, has been reported in patients on conventional therapy [4]. Until recently, thymectomy has been performed on an empiric basis in nonthymomatous MG. The first randomized clinical trial ever published (126 patients randomized with less than 5-year disease duration) favored thymectomy plus alternate day prednisone over alternative day predinsone alone, with fewer patients needing immunosuppressants and lower doses of prednisone in the thymectomized group [5]. The patients were followed up for a period over 3 years. Nonetheless, further clinical 
1. To elucidate the role of the thymus gland in myasthenia gravis.

2. To find out what initiates immunogenicity (drugs, vaccines, infections, genetic propensity, etc.) and why immunogenicity becomes chronic. This is essential for prevention and treatment purposes.

3. New biomarkers of long-term prognosis and risk of myasthenic crisis.

4. Surveillance programs for patients on immunosuppressive drugs.

5. To establish the preference of some immunosuppressants over the rest.

6. To share information among clinicians for better indication of drugs by means of appropriate registries.

7. To learn from other autoimmune diseases.

trials are needed to ascertain the subset of patients who can benefit specifically from the procedure. Moreover, the effects of tymectomy are not immediate and remissions can occur after years of the intervention. Other unanswered questions are when to perform thymectomy and what we can expect from it.

Medical treatment is based on cholinesterase inhibitors, corticosteroids and immunosuppressants. In accordance with the International Consensus Guidance [6], cholinesterase inhibitors and prednisone are safe in pregnancy and azathioprine as well. For myasthenic crisis, either intravenous immunoglobulins or plasmapheresis are an effective treatment. Long-term maintenance therapy poses some caveats. Many patients need high doses of prednisone for doing well, but the potential side effects of corticosteroids make the clinicians to substitute prednisone for immunosuppressive drugs. Azathioprine is one of the most and best tolerated immunosuppressants in MG patients, with the doses adjusted for the levels of thio-purine-methyl-tranferase. It allows the clinicians to either taper or withdraw corticosteroids. Notwithstanding the benefits of azathioprine, there is a non-negligible risk of malignancies over time, especially in patients taking it for 10 years or longer so surveillance programs are needed. For cases in which azathioprine is not tolerated or does not work, another question arises: what other immunosuppressants should be given?; cyclosporine?; tacrolimus?; mycophenolate?; Rituximab?. Given the lack of clinical trials, it would be very useful to create registries so as to share the experience among colleagues. The Myasthenia Gravis Foundation of America has created a patient registry to promote research, treatment, advocacy and public awareness of MG (www.myasthenia.org). This registry is expected to be a source of important information for clinicians and researchers.

Rituximab (anti-CD20 monoclonal antibody) has emerged as an alternative option in cases of refractory MG, with good response in some cases. The evidence of action has only based on case reports and series (169 patients treated) [7]. Predictors of response were: positivity for anti-MuSK antibodies, less severe disease and younger age. But the fear of serious complications (i.e., progressive multifocal leucoencephalopathy) limits the number of infusions.

Eculizumab is another monoclonal antibody that acts by inhibiting the terminal part of the complement cascade, which is overactive in MG. The drug is used in paroxysmal nocturnal hemoglobinuria and it has been recently approved by the European Commission and launched in Germany for refractory and generalized AChR-positive MG. Eculizumab has demonstrated to be of benefit in a Phase II small clinical trial $(n=14)$ for 16 weeks, with most patients on eculizumab showing a 3-point reduction in the quantitative MG score [8]. A posterior Phase III clinical trial has not clearly confirmed the benefit of the drug (REGAIN-study or MG-301) [9]. This trial was done in 76 hospitals in 17 countries; 125 patients with refractory MG were randomly allocated to receive either eculizumab or placebo for 26 weeks. The trial failed to demonstrate significant improvement from baseline in the daily living activities scale in the eculizumab group in comparison with placebo. A posthoc analysis yielded some benefits in determined parameters such as muscle strength and quality of life. The benefits also remained in a long-term open-label extension study (MG-302) up to 52 weeks [10]. Although there were no major side effects in these trials, the US FDA warned on the serious meningococcal and other infections, so the patients should be closely monitored. Another drawback of eculizumab is its sky high price (around US $\$ 400,000$ per year).

Despite the new developments in MG, no breakthroughs have emerged in the last decade in this field and there are several unmet needs that should be addressed in further research (see Box 1).

Financial \& competing interests disclosure

The author has no relevant affiliations or financial involvement with any organization or entity with a financial interest in or financial conflict with the subject matter or materials discussed in the manuscript. This includes employment, consultancies, honoraria, stock ownership or options, expert testimony, grants or patents received or pending, or royalties. 
No writing assistance was utilized in the production of this manuscript.

\section{References}

1. Deenen JC, Horlings CG, Verschuuren JJ, Verbeek AL, van Engelen BG. The epidemiology of neuromuscular disorders: a comprehensive overview of the literature. J. Neuromuscul. Dis. 2(1), 73-85 (2015).

2. Stergiou C, Lazaridis K, Zouvelou V et al. Titin antibodies in 'seronegative' myasthenia gravis: a new role for an old antigen. J. Neuroimmunol. 292, 108-115 (2016).

3. Cordts I, Bodart N, Hartmann K et al. Screening for lipoprotein receptor-related protein 4-, agrin- and titin-antibodies and exploring the autoimmune spectrum in myasthenia gravis. J. Neurol. 264(6), 1193-1203 (2017).

4. Modrego PJ, Arribas J. Spontaneous resolution of a mediastinal mass in a woman with myasthenia gravis. Neurologia doi:10.1016/j.nrl.2016.05.005 (2016) (Epub ahead of print).

5. Wolfe GI, Kaminski HJ, Aban IB et al. Randomized trial of thymectomy in myasthenia gravis. N. Engl. J. Med. 375(6), 511-522 (2016).

6. Sanders DB, Wolfe GI, Benatar M et al. International consensus guidance for management of myasthenia gravis: executive summary. Neurology 87(4), 419-425 (2016).

7. Tandan R, Hehir MK 2nd, Waheed W, Howard DB. Rituximab treatment of myasthenia gravis: a systematic review. Muscle Nerve 56(2), 185-196 (2017).

8. Howard JF Jr, Barohn RJ, Cutter GR et al. A randomized, double-blind, placebo-controlled Phase II study of eculizumab in patients with refractory generalized myasthenia gravis. Muscle Nerve 48(1), 76-84 (2013).

9. Howard JF Jr, Utsugisawa K, Benatar M et al. Safety and efficacy of eculizumab in antiacetylcholine receptor antibody-positive refractory generalised myasthenia gravis (REGAIN): a Phase III, randomized, double-blind, placebo-controlled, multicentre study. Lancet Neurol. 16(12), 976-986 (2017).

10. Dhillon S. Eculizumab: a review in generalized myasthenia gravis. Drugs 78(3), 367-376 (2018). 